\title{
TAKESAKI'S DUALITY FOR A NON-DEGENERATE CO-ACTION
}

YOSHIKAZU KATAYAMA

\section{Abstract.}

Let $\delta$ be a non-degenerate co-action of a locally compact group $G$ on a $\mathrm{C}^{*}$ algebra $A$. We can find an action $\hat{\delta}$ on a $\delta$-crossed product $A \times{ }_{\delta} G$ and show that a crossed product $\left(A \times{ }_{\delta} G\right) \times{ }_{\delta} G$ is isomorphic to $A \otimes C\left(L^{2}(G)\right)$ where $C\left(L^{2}(G)\right)$ is the algebra of all compact operators on $L^{2}(G)$.

$A$ and $B$ are $C^{*}$-algebras. We denote by $M(A)$ the multiplier algebra of $A$. If $A$ is a concrete $\mathrm{C}^{*}$-algebra, we may define $M(A)=\left\{a \in A^{\prime \prime} ; a b+c a \in A\right.$ for $b, c \in A\}$. Following [1] we put

$$
\tilde{M}(A \otimes B)=\{x \in M(A \otimes B) ; x(1 \otimes b)+(1 \otimes c) x \in A \otimes B \text { for } b, c \in B\},
$$

where the symbol $\otimes$ means the spatial tensor product.

Let $G$ be a locally compact group. $L^{2}(G)$ is the Hilbert space of square integrable functions on $G$ with a left Haar measure $d s$ on $G$. The left and right regular representations of $G$ on $L^{2}(G)$ are defined by

$$
\begin{aligned}
& (\lambda(s) \xi)(t)=\xi\left(s^{-1} t\right) \\
& (\varrho(s) \xi)(t)=\Delta^{-\frac{1}{2}}(s) \xi(t s)
\end{aligned}
$$

for $s, t \in G$ and $\xi \in L^{2}(G)$, where $\Delta$ is the modular function of $G$. Let $C_{r}^{*}(G)$ be the $C^{*}$-algebra generated by $\left\{\lambda(f) ; f \in L^{1}(G)\right\}$ where

$$
\lambda(f)=\int_{G} f(s) \lambda(s) d s,
$$

which is called the reduced group $C^{*}$-algebra of $G$. We define a unitary operator $W$ on $L^{2}(G \times G)$ by

$$
(W \xi)(s, t)=\xi(s, s t)
$$

for $\xi \in L^{2}(G \times G)$ and we set $\delta_{G}(x)=W^{*}(x \otimes 1) W=\operatorname{Ad} W^{*}(x \otimes 1)$ for $x \in C_{r}^{*}(G)$. Then we can show easily that $\delta_{G}$ is an isomorphism of $C_{r}^{*}(G)$ into $\tilde{M}\left(C_{r}^{*}(G) \otimes C_{r}^{*}(G)\right)$. Since $\delta_{G}\left(C_{r}^{*}(G)\right)\left(1 \otimes C_{r}^{*}(G)\right)$ generates $C_{r}^{*}(G) \otimes C_{r}^{*}(G)$, for 
each approximate identity $\left\{e_{i}\right\}$ of $C_{r}^{*}(G), \delta_{G}\left(e_{i}\right)$ converges to 1 in the strict topology of $M\left(C_{r}^{*}(G) \otimes C_{r}^{*}(G)\right)$.

Let $\theta$ be a homomorphism of $A$ into $M(B)$ satisfying that $\theta\left(u_{i}\right)$ converges to 1 in the strict topology of $M(B)$ for each approximate identity $\left\{u_{i}\right\}$ of $A$. Then $\theta$ extends uniquely to a homomorphism (also denoted by $\theta$ ) of $M(A)$ into $M(B)$ ([8, Lemme 0.2.6]). The above $\delta_{G}$ has a property

$$
\left(\delta_{G} \otimes \imath\right) \delta_{G}=\left(\imath \otimes \delta_{G}\right) \delta_{G},
$$

where $l$ is the identity map of $C_{r}^{*}(G)$ (the above $\delta_{G} \otimes \imath$ and $l \otimes \delta_{G}$ are homomorphisms on $M\left(C_{r}^{*}(G) \otimes C_{r}^{*}(G)\right)$ ).

Definition. Let $\delta$ be an isomorphism of $A$ into $\tilde{M}\left(A \otimes C_{r}^{*}(G)\right)$. The isomorphism $\delta$ is called a co-action of $G$ on $A$ if for each approximate identity $\left\{u_{i}\right\}$ of $A, \delta\left(u_{i}\right)$ converges to 1 in the strict topology of $M\left(A \otimes C_{r}^{*}(G)\right)$ and $(\delta \otimes \imath) \delta=\left(\imath \otimes \delta_{G}\right) \delta$.

We define a linear map $\delta_{u}$ by $\delta_{u}(a)=L_{u} \delta(a)$ for $u \in B_{r}(G) \equiv C_{r}^{*}(G)^{*}, a \in A$ where $L_{u}$ is the left slice map of $u$ (see [2]). Since $\delta$ is a map into $\tilde{M}\left(A \otimes C_{r}^{*}(G)\right)$, by [7, Theorem 2.1] $\delta_{u}$ is a linear map of $A$ into $A$.

Lemma 1. Let $\delta$ be a co-action of $G$ on $A$. For $x=\delta_{u}(a), a \in A$ and $u \in B_{r}(G) \cap K(G)$, we have

$$
\int_{G} \delta_{\varphi \lambda(s)^{*}}(x) \otimes \lambda(s) z d s=\delta(x)(1 \otimes \lambda(\check{\varphi}) z)
$$

for $\varphi \in B_{r}(G) \cap K(G)$ and $z \in C_{r}^{*}(G)$, where $\check{\varphi}(s)=\varphi\left(s^{-1}\right)$ and $\left\langle z, \varphi \lambda(s)^{*}\right\rangle$ $=\left\langle\lambda(s)^{*} z, \varphi\right\rangle(K(G)$ is the family of continuous functions on $G$ with compact supports).

Proof. Both functions $s \in G \rightarrow \lambda(s) z \in C_{r}^{*}(G)$ and $s \in G \rightarrow \varphi \lambda(s)^{*} \in$ $C_{r}^{*}(G)^{*}$ are norm-continuous. The integrand:

$$
s \in G \rightarrow \delta_{\varphi \lambda(s)^{*}}(x) \otimes \lambda(s) z
$$

is continuous in the norm topology of $A \otimes C_{r}^{*}(G)$, whose support is contained in a compact set $(\operatorname{supp} u) \cdot(\operatorname{supp} \varphi)^{-1}$. Hence $\int_{G} \delta_{\varphi \lambda(s)^{*}}(x) \otimes \lambda(s) z d s$ is contained in $A \otimes C_{r}^{*}(G)$. For $\omega \in A^{*}, \psi \in B_{r}(G) \cap K(G)$ and $z=\lambda(f), \in K(G)$, we have

$$
\begin{aligned}
& \left\langle\int_{G} \delta_{\varphi \lambda(s)^{*}}(x) \otimes \lambda(s) z d s, \omega \otimes \psi\right\rangle \\
= & \int_{G}\left\langle\delta(x), \omega \otimes \varphi \lambda(s)^{*}\right\rangle\langle\lambda(s) z, \psi\rangle d s,
\end{aligned}
$$


since the function

$$
s \in G \rightarrow\langle\lambda(s) z, \psi\rangle=\langle\lambda(s) \lambda(f), \psi\rangle
$$

is continuous whose support is contained in a compact set $(\operatorname{supp} \psi) \cdot(\operatorname{supp} f)^{-1}$,

$$
=\left\langle\delta(x), \omega \otimes \int_{G}\langle\lambda(s) z, \psi\rangle \varphi \lambda(s)^{*} d s\right\rangle .
$$

For $g \in L^{1}(G)$, we get

$$
\begin{aligned}
& \left\langle\lambda(g), \int_{G}\langle\lambda(s) z, \psi\rangle \varphi \lambda(s)^{*} d s\right\rangle \\
= & \int_{G}\langle\lambda(s) z, \psi\rangle\left\langle\lambda(s)^{*} \lambda(g), \varphi\right\rangle d s \\
= & \iint_{G \times G}\langle\lambda(s) z, \psi\rangle g(t)\left\langle\lambda\left(s^{-1} t\right), \varphi\right\rangle d t d s \\
= & \iint_{G \times G}\langle\lambda(t h) z, \psi\rangle g(t)\left\langle\lambda\left(h^{-1}\right), \varphi\right\rangle d t d h \\
= & \int_{G}\langle\lambda(g) \lambda(h) z, \psi\rangle\left\langle\lambda\left(h^{-1}\right), \varphi\right\rangle d h \\
= & \int_{G} \check{\varphi}(h)\langle\lambda(g) \lambda(h) z, \psi\rangle d h=\langle\lambda(g) \lambda(\check{\varphi}) z, \psi\rangle \\
= & \langle\lambda(g), \lambda(\check{\varphi}) z \psi\rangle .
\end{aligned}
$$

Therefore we have $\int_{G}\langle\lambda(s) z, \psi\rangle \varphi \lambda(s)^{*} d s=\lambda(\check{\varphi}) z \psi$. Hence

$$
(2)=\langle\delta(x), \omega \otimes \lambda(\check{\varphi}) z \psi\rangle=\langle\delta(x)(1 \otimes \lambda(\check{\varphi}) z), \omega \otimes \psi\rangle \text {. }
$$

Since $B_{r}(G) \cap K(G)$ is dense in the Fourier algebra $A(G)$ (see [2]), we obtain

$$
\int_{G} \delta_{\varphi \lambda(s)^{*}}(x) \otimes \lambda(s) z d s=\delta(x)(1 \otimes \lambda(\check{\varphi}) z)
$$

for $z=\lambda(f), f \in K(G)$. Both sides in (3) are continuous with respect to $z$. Then we have the equation (3) for all $z \in C_{r}^{*}(G)$.

Lemma 2. Let $\delta$ be as above. The closure $I(A)$ of $\left\{\delta_{\varphi}(a) ; a \in A, \varphi \in A(G)\right\}$ is a $C^{*}$-subalgebra of $A$. Moreover for $x \in I(A)$ and $z \in C_{r}^{*}(G)$ the element $\delta(x)(1 \otimes z)$ is contained in $I(A) \otimes C_{r}^{*}(G)$. 
Proof. Since $K(G) \cap A(G)$ is norm-dense in $A(G)$ and $\left\|\delta_{\varphi}\right\| \leqq\|\varphi\|$ for $\varphi \in B_{r}(G), I(A)$ is the closure of $\left\{\delta_{\varphi}(a) ; a \in A, \varphi \in K(G) \cap A(G)\right\}$. Since $A(G)$ is a regular ring (see [2]), we can find, for $\varphi_{1}, \varphi_{2} \in K(G) \cap A(G), \varphi_{3}$ in $K(G) \cap A(G)$ with $\varphi_{3} \equiv 1$ on a neighbourhood of $\left(\operatorname{supp} \varphi_{1}\right) \cdot\left(\operatorname{supp} \varphi_{2}\right)$ and $\operatorname{supp} \varphi_{1} \cup \operatorname{supp} \varphi_{2}$. Then we obtain

$$
\begin{aligned}
\delta_{\varphi_{3}}\left(\delta_{\varphi_{1}}(x) \delta_{\varphi_{2}}(y)\right) & =\delta_{\varphi_{1}}(x) \delta_{\varphi_{2}}(y) \\
\delta_{\varphi_{3}}\left(\delta_{\varphi_{1}}(x)+\delta_{\varphi_{2}}(y)\right) & =\delta_{\varphi_{1}}(x)+\delta_{\varphi_{2}}(y)
\end{aligned}
$$

for all $z, y \in A$. Therefore $I(A)$ is a $\mathrm{C}^{*}$-subalgebra of $A$. When we choose an approximate identity $\left\{\varphi_{i}\right\}$ of $L^{1}(G)$ in the set $K(G) \cap B_{r}(G)=K(G) \cap A(G)$, by the equation (1) we have

$$
\lim _{i} \int_{G} \delta_{\varphi_{i} \lambda(s)^{*}}(x) \otimes \lambda(s) z d s=\lim _{i} \delta(x)\left(1 \otimes \lambda\left(\check{\varphi}_{i}\right) z\right)=\delta(x)(1 \otimes z),
$$

that is $\delta(x)(1 \otimes z)$ is contained in $I(A) \otimes C_{r}^{*}(G)$ for $x=\delta_{u}(y)$, some $y \in A$ and $u \in K(G) \cap A(G)$. Therefore $\delta(x)(1 \otimes z)$ is contained in $I(A) \otimes C_{r}^{*}(G)$ for $x \in I(A)$.

Lemma 3. Let $\delta$ be as above. The closed subspace $\left[\delta(I(A))\left(1 \otimes C_{r}^{*}(G)\right)\right]$ generated by $\delta(I(A))\left(1 \otimes C_{r}^{*}(G)\right)$ contains $I(A) \otimes C_{r}^{*}(G)$.

Proof. Take $x=\delta_{u}(y),(y \in A, u \in A(G) \cap K(G))$, and by (1) we have, for $\varphi \in A(G) \cap K(G)$,

$$
\delta(x)(1 \otimes \lambda(\check{\varphi}))=\int_{G} \delta_{\varphi \lambda(s)^{*}}(x) \otimes \lambda(s) d s \quad \text { (in the strict topology) } .
$$

For $v \in A(G) \cap K(G)$, we obtain

$$
\begin{aligned}
\iota \otimes L_{v} & {\left[\left(\imath \otimes \delta_{G}\right)\{\delta(x)(1 \otimes \lambda(\check{\varphi}))\}\right] } \\
& =\imath \otimes L_{v}\left(\int_{G} \delta_{\varphi \lambda(s)^{*}}(x) \otimes \lambda(s) \otimes \lambda(s) d s\right) \\
& =\int_{G} v(s) \delta_{\varphi \lambda(s)^{*}}(x) \otimes \lambda(s) d s .
\end{aligned}
$$

On the other hand, we get, for $\omega_{1} \in A^{*}, \omega_{2} \in C_{r}^{*}(G)^{*}$

$$
\begin{aligned}
& \left\langle\imath \otimes L_{v}\left[\left(\iota \otimes \delta_{G}\right)(\delta(x)(1 \otimes \lambda(\check{\varphi})))\right], \omega_{1} \otimes \omega_{2}\right\rangle \\
= & \left\langle\left(\left(\imath \otimes \delta_{G}\right) \delta(x)\right)\left(1 \otimes \delta_{G}(\lambda(\check{\varphi}))\right), \omega_{1} \otimes \omega_{2} \otimes v\right\rangle \\
= & \left\langle(\delta \otimes \imath) \delta(x), \omega_{1} \otimes\left(\int_{G} \varphi(s) \lambda(s) \otimes \lambda(s) d s\right)\left(\omega_{2} \otimes v\right)\right\rangle
\end{aligned}
$$




$$
\begin{aligned}
& =\int_{G} \check{\varphi}(s)\left\langle(\delta \otimes \imath) \delta(x), \omega_{1} \otimes \lambda(s) \omega_{2} \otimes \lambda(s) v\right\rangle d s \\
& =\int_{G} \check{\varphi}(s)\left\langle\delta\left(\delta_{\lambda(s) v}(x)\right)(1 \otimes \lambda(s)), \omega_{1} \otimes \omega_{2}\right\rangle d s \\
& =\left\langle\int_{G} \check{\varphi}(s) \delta\left(\delta_{\lambda(s) v}(x)\right)(1 \otimes \lambda(s)) d s, \omega_{1} \otimes \omega_{2}\right\rangle .
\end{aligned}
$$

Therefore

$$
\begin{gathered}
l \otimes L_{v}\left[\left(\imath \otimes \delta_{G}\right)(\delta(x)(1 \otimes \lambda(\check{\varphi})))\right] \\
=\int_{G} \check{\varphi}(s) \delta\left(\delta_{\lambda(s) v}(x)\right)(1 \otimes \lambda(s)) d s=\int_{G} v(s) \delta_{\varphi \lambda(s)^{*}}(x) \otimes \lambda(s) d s .
\end{gathered}
$$

For $z \in C_{r}^{*}(G)$ we obtain,

$$
\int_{G} \check{\varphi}(s) \delta\left(\delta_{\lambda(s) v}(x)\right)(1 \otimes \lambda(s) z) d s=\int_{G} v(s) \delta_{\varphi \lambda(s)^{*}}(x) \otimes \lambda(s) z d s .
$$

Since the integrands in the above equation are norm-continuous,

$$
\lim _{v} \int_{G} \check{\varphi}(s) \delta\left(\delta_{\lambda(s) v}(x)\right)(1 \otimes \lambda(s) z) d s=\delta_{\varphi}(x) \otimes z
$$

in the norm topology, when the measure $v(s) d s$ tends to a Dirac measure at the identity of $G$. Then $\left[\delta(I(A))\left(1 \otimes C_{r}^{*}(G)\right)\right]$ contains $I(A) \otimes C_{r}^{*}(G)$.

Remark. The restriction $\left.\delta\right|_{I(A)}$ of $\delta$ to $I(A)$ is a co-action of $G$ on $I(A)$.

Lemma 4. Let $\delta$ be as above. The closed linear $\operatorname{span}\left[\delta(A)\left(1 \otimes C_{r}^{*}(G)\right)\right]$ is coincided with $I(A) \otimes C_{r}^{*}(G)$.

Proof. Without the condition $x=\delta_{u}(y)$ in the proof of the former equality in (4), we have, for $v, \varphi \in A(G) \cap K(G), x \in A$,

$$
\imath \otimes\left(\delta_{G}\right)_{v}(\delta(x)(1 \otimes \lambda(\check{\varphi})))=\int_{G} \check{\varphi}(s) \delta\left(\delta_{\lambda(s) v}(x)\right)(1 \otimes \lambda(s)) d s .
$$

Since $A \otimes C_{r}^{*}(G)$ contains $\delta(x)(1 \otimes \lambda(\check{\varphi}))$, the norm closure of $\left\{\left(\imath \otimes\left(\delta_{G}\right)_{v}\right)(\delta(x)(1 \otimes \lambda(\check{\varphi}))) ; v \in A(G) \cap K(G)\right\}$ contains $\delta(x)(1 \otimes \lambda(\check{\varphi}))$. The norm closure of

$$
\left\{\int_{G} \check{\varphi}(s) \delta(\lambda(s) v(x))(1 \otimes \lambda(s) z) d s ; v \in A(G) \cap K(G)\right\}
$$


contains $\delta(x)(1 \otimes \lambda(\check{\varphi}) z), \quad\left(z \in C_{r}^{*}(G)\right)$. Since the element $\delta_{\lambda(s) v}(x)$ is in $I(A)$, Lemma 2 implies that $\int_{G} \check{\varphi}(s) \delta\left(\delta_{\lambda(s) v}(x)\right)(1 \otimes \lambda(s) z) d s$ is in $I(A) \otimes C_{r}^{*}(G)$, that is

$$
\delta(x)(1 \otimes \lambda(\check{\varphi}) z) \in I(A) \otimes C_{r}^{*}(G) .
$$

By taking $\varphi$ as an approximate identity of $L^{1}(G)$, we get $\delta(x)(1 \otimes z) \in I(A) \otimes C_{r}^{*}(G)$ for $x \in A$ and $z \in C_{r}^{*}(G)$. By Lemma 3, we have $\left[\delta(A)\left(1 \otimes C_{r}^{*}(G)\right)\right]=I(A) \otimes C_{r}^{*}(G)$.

THEOREM 5. Let $\delta$ be as above. The following statements are equivalent.

(i) $A=I(A)$,

(ii) $\left[\delta(A)\left(1 \otimes C_{r}^{*}(G)\right)\right]=A \otimes C_{r}^{*}(G)$,

(iii) $\left[\delta(A)\left(1 \otimes C\left(L^{2}(G)\right)\right)\right]=A \otimes C\left(L^{2}(G)\right)$,

(iv) $\delta$ is non-degenerate in Landstad's sence, i.e. for each non-zero linear functional $\omega$ in $A^{*}$, we can find $u \in B_{r}(G)$ with $(\omega \otimes u) \delta \neq 0$.

Proof. The equivalence of (i) and (ii) follows from Lemma 4. Since $C_{r}^{*}(G) \cdot C_{0}(G)$ generates $C\left(L^{2}(G)\right.$ ), we have (ii) $\Rightarrow$ (iii). We shall prove (iii) $\Rightarrow$ (i). We define a rank one operator $\xi \otimes \eta^{c}$ with $\left(\xi \otimes \eta^{c}\right)(\zeta)=\langle\zeta, \eta\rangle \xi$ for $\xi, \eta$ and $\zeta \in L^{2}(G)$. For $\xi_{i}, \eta_{i}(i=1,2), \xi, \eta \in L^{2}(G)$ and elements $p, q$ of the universal Hilbert space for $A$, we have

$$
\begin{aligned}
& \left\langle\left\{1 \otimes\left(\xi_{1} \otimes \eta_{1}^{c}\right)\right\} \delta(a)\left\{1 \otimes\left(\xi_{2} \otimes \eta_{2}^{c}\right)\right\} p \otimes \xi, q \otimes \eta\right\rangle \\
= & \left\langle\delta(a)\left(p \otimes\left\langle\xi, \eta_{2}\right\rangle \xi_{2}\right), q \otimes\left\langle\eta, \xi_{1}\right\rangle \eta_{1}\right\rangle \\
= & \left\langle\delta(a)\left(p \otimes \xi_{2}\right), q \otimes \eta_{1}\right\rangle\left\langle\xi, \eta_{2}\right\rangle \overline{\left\langle\eta, \xi_{1}\right\rangle} \\
= & \left\langle\delta_{\omega_{\xi_{2}, \eta_{1}}}(a) p, q\right\rangle\left\langle\left\langle\xi, \eta_{2}\right\rangle \xi_{1}, \eta\right\rangle
\end{aligned}
$$

where

$$
\begin{aligned}
& \omega_{\xi_{2} \eta_{1}}(z)=\left\langle z \xi_{2}, \eta_{1}\right\rangle \\
& =\left\langle\delta_{\omega_{\xi_{2}, \eta_{1}}}(a) \otimes\left(\xi_{1} \otimes \eta_{2}^{c}\right)(p \otimes \xi), q \otimes \eta\right\rangle .
\end{aligned}
$$

Then

$$
\left[1 \otimes\left(\xi_{1} \otimes \eta_{1}^{c}\right)\right] \delta(a)\left[1 \otimes\left(\xi_{2} \otimes \eta_{2}^{c}\right)\right]=\delta_{\omega_{\xi_{2}, \eta_{1}}}(a) \otimes\left(\xi_{1} \otimes \eta_{2}^{c}\right)
$$

Since the family of finite rank operators on $L^{2}(G)$ generates $C\left(L^{2}(G)\right)$, $\delta(A)\left(1 \otimes C\left(L^{2}(G)\right)\right)$ is contained in $I(A) \otimes C\left(L^{2}(G)\right)$. Then by (iii), we have $A \otimes C\left(L^{2}(G)\right)=I(A) \otimes C\left(L^{2}(G)\right)$, which implies $A=I(A)$. If (ii) holds, for non zero functionals $\omega$ in $A^{*}$ and $u$ in $B_{r}(G)$, we can find $a$ in $A$ with $(\omega \otimes a u) \delta \neq 0$. Suppose that $I(A)$ is a proper $C^{*}$-subalgebra of $A$. We can find a non zero linear functional $\omega$ in $A^{*}$ with $\omega(I(A))=0$. Then it follows from Lemma 4 and 
[7, Theorem 2.1] that $(\omega \otimes u) \delta=0$ for all $u \in B_{r}(G)$, which is a contradiction with non-degeneracy of $\delta$.

It is found in [4, Lemma 3.8] that a co-action $\delta$ of a discrete or amenable group $G$ is automatically non-degenerate. Also a canonical co-action on a reduced crossed product for a $\mathrm{C}^{*}$-dynamical system is automatically nondegenerate. The author has been unable to prove the automatical nondegeneracy of $\delta$ for arbitrary locally compact group. For convenience of readers, we prove the automatical non-degeneracy for a discrete or amenable group. We prove the condition (i) in Theorem 5 in a slight different way.

Proposition 6 ([4]). Let $G$ be a discrete or amenable group. A co-action $\delta$ of $G$ on $A$ is automatically non-degenerate.

Proof. By [7, Theorem 2.1], for $u \in B_{r}(G)$, we find $a$ in $A$ and $v \in B_{r}(G)$ with $u=a v$. Then we have

$$
\delta_{u}(g)=\delta_{a v}(x)=L_{v}(\delta(x)(1 \otimes a))
$$

in $I(A)$ by Lemma 4 . We have

$$
\left\{\begin{aligned}
\delta\left(\delta_{u}(x)\right) & =\delta L_{u}(\delta(x))=\left(\imath \otimes L_{u}\right)(\delta \otimes \imath) \delta(x) \\
& =\imath \otimes L_{u}\left(\left(\iota \otimes \delta_{G}\right)(\delta(x))\right)=\imath \otimes\left(\delta_{G}\right)_{u}(\delta(x)) .
\end{aligned}\right.
$$

Suppose that $G$ is amenable, we take the identity $u_{1}(s) \equiv 1$ in $B_{r}(G)=B(G)$. Since $\imath \otimes\left(\delta_{G}\right)_{u_{1}}$ is an identity map of $M\left(A \otimes C_{r}^{*}(G)\right)$, we have $\delta\left(\delta_{u_{1}}(x)\right)=\delta(x)$ for $x \in A$, which implies $x=\delta_{u_{1}}(x) \in I(A)$ by the injectivity of $\delta$. Suppose that $G$ is discrete. Then $\delta(x)$ is contained in $A \otimes C_{r}^{*}(G)$. Therefore it is easy to prove that the closure of $\left\{l \otimes\left(\delta_{G}\right)_{u}(\delta(x)) ; u \in B_{r}(G)\right\}$ contains $\delta(x)$. By $(5), \delta(I(A))$ contains $\delta(x)$ for $x \in A$, that is $x \in I(A)$. In the both cases we have $A=I(A)$.

Let $C^{*}(G)$ be the envelopping $C^{*}$-algebra of $L^{1}(G)$, and $U$ be the universal representation of $G$. We can define an isomorphism $\overline{\delta_{G}}$ of $C^{*}(G)$ into $\tilde{M}\left(C^{*}(G) \otimes C^{*}(G)\right)$ such that

$$
\begin{aligned}
\overline{\delta_{G}}(U(f)) & =\overline{\delta_{G}}\left(\int_{G} f(s) U(s) d s\right) \\
& =\int_{G} f(s) U(s) \otimes U(s) d s
\end{aligned}
$$

for $f \in L^{1}(G)$. Moreover $\left(\imath \otimes \overline{\delta_{G}}\right) \overline{\delta_{G}}=\left(\overline{\delta_{G}} \otimes \imath\right) \overline{\delta_{G}}$ and

$$
\left[\overline{\delta_{G}}\left(C^{*}(G)\right)\left(1 \otimes C^{*}(G)\right)\right]=C^{*}(G) \otimes C^{*}(G)
$$

(see [3, Theorem 3.9]). 
Let $\delta$ be an injective homomorphism of $A$ into $\tilde{M}\left(A \otimes C^{*}(G)\right)$ and $\delta\left(e_{n}\right)$ convergens 1 in the strict topology of $A \otimes C^{*}(G)$ for each approximate identity $\left\{e_{n}\right\}$ of $A$ and $(\delta \otimes \imath) \delta=\left(\imath \otimes \overline{\delta_{G}}\right) \delta$. Let $\pi$ be a canonical homomorphism of $C^{*}(G)$ onto $C_{r}^{*}(G)$. Note that $\delta$ automatically satisfies the statements in Theorem 5 by the same proof as in the case of an amenable group $G$ (Proposition 6). Set

$$
\delta^{1}(x)=(l \otimes \pi) \delta(x) \quad \text { for } x \in A .
$$

Since $\delta^{1}$ is not in general injective, set

$$
I=\operatorname{Ker} \delta^{1} \quad \text { and } \quad \delta^{r}(\theta(x))=(\theta \otimes \imath) \delta^{1}(x) \quad \text { for } x \in A,
$$

where $\theta$ is a canonical homomorphism of $A$ onto $A / I$.

Proposition 7. The map $\delta^{r}$ is a non-degenerate co-action of $G$ on $A / I$.

Proof. For $f \in L^{1}(G)$ and $x \in A$, we have

$$
\begin{aligned}
& \delta^{r}(\theta(x))(1 \otimes \lambda(f))=(\theta \otimes \imath) \delta^{1}(x)(1 \otimes \lambda(f)) \\
& =[(\theta \otimes \imath)(\imath \otimes \pi) \delta(x)](1 \otimes \lambda(f))=\theta \otimes \pi(\delta(x)(1 \otimes U(f))),
\end{aligned}
$$

because of $\pi(U(f))=\lambda(f)$. Then $\delta^{r}(\theta(x))(1 \otimes z)$ is contained in $A / I \otimes C_{r .(G)}^{*}(G)$ for $x \in A$ and $z \in C_{r}^{*}(G)$. Suppose $\delta^{r}(\theta(x))=0(x \in A)$. Then for $\omega \in C_{r}^{*}(G)^{*}$, we have

$$
\begin{aligned}
0 & =L_{\omega}\left(\delta^{r}(\theta(x))\right)=L_{\omega}\left((\theta \otimes \imath) \delta^{1}(x)\right) \\
& =\theta\left(L_{\omega} \delta^{1}(x)\right)=\theta\left(\delta_{\omega}^{1}(x)\right) .
\end{aligned}
$$

Therefore $\delta_{\omega}^{1}(x)$ is contained in $I$. Since

$$
\left(l \otimes L_{\omega}\right)\left(\delta^{1} \otimes \imath\right) \delta^{1}(x)=\delta^{1}\left(\delta_{\omega}^{1}(x)\right)=0 \quad \text { for } \omega \in C_{r}^{*}(G)^{*},
$$

we have $\left(\delta^{1} \otimes \imath\right) \delta^{1}(x)=0$. Since

$$
\begin{aligned}
\left(\delta^{1} \otimes \imath\right) \delta^{1} & =[l \otimes \pi \otimes \imath)(\delta \otimes \imath)][(\imath \otimes \pi) \delta] \\
& =(l \otimes \pi \otimes \pi)(\delta \otimes \imath) \delta=(l \otimes \pi \otimes \pi)\left(\imath \otimes \overline{\delta_{G}}\right) \delta \\
& =\left(l \otimes \delta_{G}\right)(\imath \otimes \pi) \delta=\left(l \otimes \delta_{G}\right) \delta^{1}
\end{aligned}
$$

because of $(\pi \otimes \pi) \overline{\delta_{G}}=\delta_{G} \circ \pi$, then we obtain $\left(l \otimes \delta_{G}\right) \delta^{1}(x)=0$. Since $\left(l \otimes \delta_{G}\right)$ is an isomorphism of $\tilde{M}\left(A \otimes C_{r}^{*}(G)\right)$ (see [1, Proposition 2.4]), we get $\delta^{1}(x)=0$, that is $\delta^{r}$ is an isomorphism of $A / I$. We have, on $A$,

$$
\begin{aligned}
\left(\delta^{r} \otimes \imath\right) \delta^{r}(\theta(x)) & =\left[\left((\theta \otimes \imath) \delta^{1}\right) \otimes \imath\right](\theta \otimes \imath) \delta^{1}(x) \\
& =\left\{\left[(\theta \otimes \imath) \delta^{1} \theta\right] \otimes \imath\right\} \delta^{1}(x)=\left\{(\theta \otimes \imath) \delta^{1} \otimes \imath\right\} \delta^{1}(x)
\end{aligned}
$$




$$
\begin{aligned}
& =(\theta \otimes \imath \otimes \imath)\left(\delta^{1} \otimes \imath\right) \delta^{1}(x)=(\theta \otimes \imath \otimes \imath)\left(\imath \otimes \delta_{G}\right) \delta^{1}(x) \\
& =\left(\imath \otimes \delta_{G}\right)(\theta \otimes \imath) \delta^{1}(x)=\left(\imath \otimes \delta_{G}\right) \delta^{r}(\theta(x)) .
\end{aligned}
$$

Since $\delta\left(e_{n}\right)$ converges to 1 in the strict topology of $M\left(A \otimes C^{*}(G)\right)$ for each approximate identity $\left\{e_{n}\right\}$ of $A$, it follows from [8, Lemme 0.2.6] that $\delta^{r}$ has the same property for $A / I$. Then we have proved that $\delta^{r}$ is a co-action of $G$ on $A / I$. Also $\delta_{u}^{r}(\theta(x))=\theta\left(\delta_{u}(x)\right)$ for $x \in A$ and $u \bar{\epsilon} A(G)$ and by the same proof as in the case of an amenable group $G$ (Proposition 6), $A$ is generated by $\left\{\delta_{u}(x)\right.$; $u \in A(G), x \in A\}$. Therefore $\left\{\delta_{u}^{r}(x) ; u \in A(G), x \in A / I\right\}$ generated $A / I$, that is $\delta^{r}$ is non-degenerate.

The isomorphism $\delta$ of $A$ into $\tilde{M}\left(A \otimes C^{*}(G)\right)$ (respectively $\tilde{M}\left(A \otimes C_{r}^{*}(G)\right)$ ) satisfying $(\delta \otimes \imath) \delta=\left(l \otimes \overline{\delta_{G}}\right) \delta$ (respectively $\left.(\delta \otimes \imath) \delta=\left(\imath \otimes \delta_{G}\right) \delta\right)$ is related with crossed product (respectively reduced crossed product).

Before we prove Takesaki's duality for a co-action, we need some notations and definitions. And we note that the discussion which we make below is the same which Landstad [5], Nakagami and Takesaki [6] and Van Heeswijck [9] do.

Let $\delta$ be a co-action of $G$ on $A$ and let $C_{0}(G)$ be the family of continuous functions on $G$ vanishing at infinity. The crossed product $A \times{ }_{\delta} G$ by $\delta$ is the $\mathrm{C}^{*}$ algebra generated by $\delta(A)\left(1 \otimes C_{0}(G)\right)$ in the full operator algebra $B\left(L^{2}(G, \mathscr{H})\right)$ ( $\mathscr{H}$ is the universal Hilbert space for $A$ and $C_{0}(G)$ acts as multiplication on $\left.L^{2}(G)\right)$. Let $V$ be a unitary operator on $L^{2}(G \times G, \mathscr{H})$ satisfying

$$
(V \xi)(s, t)=\Delta(t)^{\frac{1}{2}} \xi\left(s t^{-1}, t\right)
$$

for $\xi \in L^{2}(G \times G, \mathscr{H})$ and $\Delta$ is the modular function of $G$. Set a dual action $\hat{\delta}$ of $G$,

$$
\hat{\delta}(x)=V(x \otimes 1) V^{*}
$$

for $x \in A \times{ }_{\delta} G$. Then $\hat{\delta}(\delta(x))=\delta(x) \otimes 1 \quad(x \in A) \quad$ and $\hat{\delta}(1 \otimes f)=1 \otimes \alpha_{G}(f)$ $\left(f \in C_{0}(G)\right)$, where

$$
\alpha_{G}(f)(s, t)=f\left(s t^{-1}\right) .
$$

Therefore $\hat{\delta}$ is an isomorphism of $A \times{ }_{\delta} G$ into $\tilde{M}\left(A \times_{\delta} G \otimes C_{0}(G)\right)$ such that $\hat{\delta}\left(e_{n}\right)$ converges to 1 in the strict topology of $M\left(A \times{ }_{\delta} G \otimes C_{0}(G)\right)$ for each approximate identity $\left\{e_{n}\right\}$ of $A \times{ }_{\delta} G$ and $(\hat{\delta} \otimes \imath) \hat{\delta}=\left(l \otimes \alpha_{G}\right) \hat{\delta}$. The crossed product $\left(A \times{ }_{\delta} G\right) \times{ }_{\delta} G$ by the action $\hat{\delta}$ is the $\mathrm{C}^{*}$-algebra generated by $\hat{\delta}\left(A \times{ }_{\delta} G\right)\left(1 \otimes 1 \otimes C_{r}^{*}(G)\right)$. Set a co-action $\hat{\delta}$ of $G$ on $\left(A \times{ }_{\delta} G\right) \times{ }_{\delta} G$,

$$
\hat{\delta}(x)=\left(1 \otimes 1 \otimes W^{*}\right)(x \otimes 1)(1 \otimes 1 \otimes W)
$$

for $x \in\left(A \times{ }_{\delta .} G\right) \times{ }_{\delta} G$. Then $\hat{\delta}$ is easily proved to be a non-degenerate co-action of $G$. 
THEOREM 8. Let $\delta$ be a non-degenerate co-action of $G$ on $A$. The $\mathrm{C}^{*}$-algebra $\left(A \times{ }_{\delta} G\right) \times{ }_{\delta} G$ is isomorphic to $A \otimes C\left(L^{2}(G)\right)$, moreover its isomorphism transfers $\hat{\delta}$ to $\tilde{\delta}$, where

$$
\tilde{\delta}(x)=(1 \otimes W)[(\iota \otimes \sigma)(\delta \otimes \imath)(x))] 1 \otimes W^{*}
$$

and $\sigma$ is a flip map of $C_{r}^{*}(G) \otimes C\left(L^{2}(G)\right)$ onto $C\left(L^{2}(G)\right) \otimes C_{r}^{*}(G)$.

Proof. Let $D$ be the $C^{*}$-algebra generated by $S(1 \otimes W)\left(\left(1 \otimes W^{*}\right)(\delta(A) \otimes 1)(1 \otimes W)\left(1 \otimes 1 \otimes C\left(L^{2}(G)\right)\right)\right)\left(1 \otimes W^{*}\right) S^{*}$, where $S$ is a unitary operator defined by

$$
(S \xi)(s, t)=\Delta(t)^{-\frac{1}{2}} \xi\left(s, t^{-1}\right) \quad\left(\xi \in L^{2}(G \times G, \mathscr{H})\right) .
$$

Then

$$
\begin{aligned}
\left(1 \otimes W^{*}\right)(\delta(A) \otimes 1)(1 \otimes W)\left(1 \otimes 1 \otimes C\left(L^{2}(G)\right)\right) \\
\quad=\left(\imath \otimes \delta_{G}\right) \delta(A)\left(1 \otimes 1 \otimes C\left(L^{2}(G)\right)\right) \\
=(\delta \otimes \imath) \delta(A)\left(1 \otimes 1 \otimes C\left(L^{2}(G)\right)\right) \\
=(\delta \otimes \imath)\left(\delta(A)\left(1 \otimes C\left(L^{2}(G)\right)\right)\right)
\end{aligned}
$$

Since $\delta$ is non-degenerate, by Theorem 5 (iii), $\delta(A)\left(1 \otimes C\left(L^{2}(G)\right)\right)$ generates $A \otimes C\left(L^{2}(G)\right)$. Then $D$ is isomorphic to $A \otimes C\left(L^{2}(G)\right)$. Therefore we have only to prove that $D$ coincides $\left(A \times{ }_{\delta} G\right) \times{ }_{\delta} G$. We prove easily the following facts:

$$
\left\{\begin{array}{lc}
S(\delta(a) \otimes 1) S^{*}=\delta(a) \otimes 1 & (a \in A) \\
S(1 \otimes W)(1 \otimes 1 \otimes v(g))\left(1 \otimes W^{*}\right) S^{*}=1 \otimes 1 \otimes \lambda(g) & \left(g \in L^{1}(G)\right) \\
\text { where } v \text { is the right regular representation of } G & \\
S(1 \otimes W)(1 \otimes 1 \otimes f)\left(1 \otimes W^{*}\right) S^{*}=1 \otimes \alpha_{G}(f) & \left(f \in C_{0}(G)\right) \\
C\left(L^{2}(G)\right) \text { is generated by }\left\{f \cdot v(g) ; f \in C_{0}(G), g \in L^{1}(G)\right\} .
\end{array}\right.
$$

By extending $\tilde{\delta}$ and $\hat{\delta}$ to their multipliers, we have

$$
\begin{cases}\tilde{\delta}(\delta(a))=\delta(a) \otimes 1 & (a \in A) \\ \tilde{\delta}(1 \otimes f)=1 \otimes f \otimes 1 & \left(f \in C_{0}(G)\right) \\ \tilde{\delta}(v(g))=\int_{G} g(s) v(s) \otimes v(s) d s & \left(g \in L^{1}(G)\right)\end{cases}
$$

and 
(8)

$$
\begin{cases}\hat{\delta}(\delta(a) \otimes 1)=\delta(a) \otimes 1 \otimes 1 & (a \in A) \\ \hat{\delta}\left(1 \otimes \alpha_{G}(f)\right)=1 \otimes \alpha_{G}(f) \otimes 1 & \left(f \in C_{0}(G)\right) \\ \hat{\delta}(1 \otimes 1 \otimes \lambda(g))=\int_{G} g(s)(1 \otimes 1 \otimes \lambda(s) \otimes \lambda(s)) d s & \left(g \in L^{1}(G)\right) .\end{cases}
$$

By (6), $D$ is isomorphic to $\left(A \times{ }_{\delta} G\right) \times{ }_{\hat{\delta}} G$. By (7), (8) its isomorphism transfers $\hat{\delta}$ to $\tilde{\delta}$.

When $G$ is a discrete or amenable group, Takesaki's duality by co-action of $G$ holds true without non-degeneracy of $\delta$. If $G$ is compact, Landstad has already solved it in [5, Theorem 3].

\section{REFERENCES}

1. C. A. Akemann, G. K. Pedersen, and J. Tomiyama, Multipliers of $\mathrm{C}^{*}$-algebras, J. Funct. Anal. 13 (1973), 277-301.

2. P. Eymard, L'algebre de Fourier d'un groupe localement compact, Bull. Soc. Math. France 92 (1964), 181-236.

3. V. M. Iorio, Hopf-C*-algebras and locally compact groups, Pacific J. Math. 87 (1980), 75-96.

4. M. B. Landstad, Duality theory for covariant systems, Trans. Amer. Math. Soc. 248 (1979), 223269.

5. M. B. Landstad, Duality for dual C*-covariance algebras over compact groups, preprint, University of Trondheim, 1978.

6. Y. Nakagami and M. Takesaki, Duality for crossed products of von Neumann algebras (Lecture Notes in Math. 731), Springer-Verlag, Berlin - Heidelberg - New York, 1979.

7. D. C. Taylor, The strict topology for double centralizer algebras, Trans. Amer. Math. Soc. 150 (1970), 633-643.

8. J. M. Vallin, $\mathrm{C}^{*}$-algebres de Hopf spatiales $\mathrm{C}^{*}$-algebres de $\mathrm{Kac}$, preprint, Université d'Orléans.

9. L. Van Heeswijck, Duality in the theory of crossed products, Math. Scand. 44 (1979), 313-329.

OSAKA UNIVERSITY

FACULTY OF ENGINEERING SCIENCE

TOYONAKA, OSAKA

JAPAN 